\title{
Major perineal tears in barnsley; a study/review of 6 I cases in the biennal period of 2003-2004
}

\begin{abstract}
Aim: The objective of this retrospective study and systematic review was to evaluate the cases of 61 women treated for major perineal tears sustained during childbirth, over a 2 year period of 2003 to 2004, in a large District General Hospital in the United Kingdom. I aimed to assess our practice and then compare it with the available evidence-based standard stated in the Green-top guidelines 29 and 23, of the Royal College of Obstetricians and Gynaecologists (RCOG), UK.
\end{abstract}

Methods: This is a retrospective study of the case notes of 61 women who had vaginal deliveries and in the process sustained major perineal trauma between 2003 and 2004 at the Labour Ward/Delivery Suite of the Department of Obstetrics and Gynaecology, Barnsley Hospitals NHS Foundation Trust. The study involved a well-defined systematic review of case notes of these women who gave birth during the defined time frame, and who sustained major perineal tears that were managed during the same time frame.

Results: The total number of deliveries in 2003 was 2255 and vaginal deliveries accounted for 1771 of these. The total number of deliveries in 2004 was 2329 and vaginal deliveries accounted for 1827 of these. There were 53 cases of third degree tears and 8 cases of fourth degree tears over this 2 year period. The incidence of major perineal tears for 2003 was $1.8 \%$ and for 2004 was $1.56 \%$, which were all within the national average of $0.6-9.0 \%$. The continence rate achieved around the first 6 week period following primary repair was $84 \%$. According to the United Kingdom Census in 2001, the total number of women in Barnsley was 111,977 , the total number of women in the child bearing age group of 15 to 49 was 51,041.Thus the prevalence of perineal trauma in Barnsley was about 6.4/10,000 women $(32 / 51,041)$ for 2003 and about 5.8/10,000 women $(29 / 51,041)$ for 2004.

Conclusion: The incidence of major perineal trauma is for 2003 was $1.8 \%$ (cf $0.6-9 \%$ ) and the incidence for 2004 was $1.56 \%$ (cf $0.6-9 \%$ ). Our figures are clearly within the national average. However, there was need for improvement in the classification of perineal injury, surgical management and training, as highlighted in the study.

Keywords: pregnancy, labour, vaginal birth, major perineal tears, barnsley
Volume 3 Issue I - 2015

\section{Anthony Emeka Madu}

Acting Specialist Registrar, Obstetrics and Gynaecology, UK

Correspondence: Anthony Emeka Madu, Acting Specialist Registrar, Obstetrics and Gynaecology, Barnsley Hospitals NHS, Foundation Trust, Barnsley, South Yorkshire, United Kingdom, Tel 44793 1626315, Email:emymadu@yahoo.co.uk

Received: July 24, 20I5 | Published: November 2, 2015

\section{Introduction}

My aimed was to carefully assess the management of 61 women who sustained major perineal trauma following vaginal delivery from 2003 to 2004 at Barnsley Hospital NHS Foundation Trust. Additionally, I aimed to compare the management of these women with the standard guidelines, ${ }^{1,3}$ to calculate the incidence/prevalence of major perineal tears in Barnsley Hospital NHS Foundation Trust and compare it with the national average.

As part of the study, I also aimed to highlight the areas the unit met the standard practice and areas of shortfalls in the management, and to make recommendations which could, if approved, form the basis of any future modification or review of the practice/protocol in the unit.

Major perineal tears (3rd and 4th degrees) involve the disruption of the anal sphincters and the rectal mucosa respectively. These occur in $0.6-9.0 \%$ of vaginal deliveries. ${ }^{1}$ However, with the advent of endoanal ultrasound, occult damage to the anal sphincter has been found to occur in $36 \%$ of women following vaginal delivery. ${ }^{1}$ Anal incontinence is defined as involuntary loss or passage of faeces, flatus or urge incontinence that is compromising the quality of life of the patient.

\section{Materials and methods}

This is a retrospective study and systematic review of the case notes of 61 women who had a vaginal delivery and sustained major perineal trauma between 2003 and 2004 at the Labour ward/Delivery suite of the Department of Obstetrics and Gynaecology, Barnsley Hospitals NHS Foundation Trust. The records of patients were retrieved from electronic data base. RCOG guidelines ${ }^{1-3}$ and other studies from an extensive literature search were retrieved and recruited. The previous study pro forma was systematically reviewed, modified and updated to be consistent with the standard practice with which to compare our practice with.

A clear time table was set for the collation of data, review of proforma, review of the first 5 case notes, review of the rest of the case notes, analysis of practice/results and date for presentation. Case notes of these patients were systematically and carefully reviewed twice to ensure consistency in assessing the management these women received, and in comparing it with the standard practice represented by the draft pro forma. There was close co-operation with the Clinical Audit Department throughout the study.

All the stages of evolution of a clinical audit namely; stated aim, setting the standards, observing current practice (by review of the case notes), comparing our performance with targets, implementing changes and evaluation were met at the appropriate scheduled time scale on the study rota. 


\section{Study proforma and setting of the standards (Audit Proforma)}

The proforma was prepared in accordance with the recommended evidence-based guidelines. Other points to be highlighted as in the guidelines are as follows:

\section{Classification of third degree tears should be consistent, uniform and reproducible namely:}
a. 3a: less than $50 \%$ of external anal sphincter thickness torn.
b. 3 b: more than $50 \%$ of external anal sphincter thickness torn.
c. 3c: internal anal sphincter torn.

Method of repair: there was no reliable clinical evidence to show that one technique is superior to the other, ${ }^{4}$ type $1 \mathrm{~b}$ evidence. However, some studies suggested the overlap technique was superior to end-toend (approximation) technique in reducing anal incontinence by $41 \%$ versus $8 \%$ in favour of the overlap method. ${ }^{1}$

Place of repair and anaesthesia: Type IV evidence showed that performing the repair in theatre under general/regional anaesthesia was associated with better outcomes. Good light source; an assistant to help; relaxation of sphincters under regional or general anaesthesia are some of the advantages of repair in the theatre.

Monofilament suture material e.g. polydioxanone (PDS) was associated with less infection and better sphincter function, because it has longer half-life than catgut and braided polyglactin (vicryl). There was no cochrane review or randomised control trial to assess best suture material available at the time of study. The use of fine sutures e.g. PDS 3.0 was associated with less discomfort and irritation (type IV evidence).

Type IV evidence suggested the use of intra-operative and post-operative broad spectrum antibiotic was associated with less postoperative infection and wound dehiscence. Inclusion of metronidazole was advisable to cover the possible anaerobic contamination from faecal matter. The use of postoperative laxatives/ stool softners (lactulose) and bulking agent (fybogel) were associated with less wound dehiscence.

Follow-up at 6-12 weeks post-delivery in a Gynaecology clinic with interest in anorectal function or a Colorectal clinic is advisable. Endoanal ultrasound/anorectal manometry should be offered to symptomatic women. The latter should also be referred for consideration for secondary repair of the sphincter. There were no Cochrane reviews or randomised control trials to suggest the best method of follow-up at the time of the study. However, the author recognised the fact that symptoms of anal incontinence can appear several months following primary or secondary repair.

Counselling on future delivery: vaginal delivery may worsen anal sphincter symptoms in $17-24 \%^{1}$ of women after a second vaginal delivery. If pregnant and symptomatic or the endoanal scan or anorectal manometry is abnormal, the option of caesarean section must be discussed with the woman. At the time of the study, there were no Cochrane reviews or randomised control trials to suggest best mode of delivery after the repair of major perineal tears for women who are totally asymptomatic.

The risk management and medico-legal aspects involve documentation of the anatomical structures involved; method used in the repair; sutures materials used and stating, if true, that instruments, swabs and needles were accounted for after the repair. The woman should be counselled and given detailed explanation of the nature of injury, aftercare, prognosis and the need for a follow-up. Appropriate documentation should be made regarding the explanation given to her, her aftercare and follow-up.

\section{Auditable standards ${ }^{\prime}$}

i. Use of agreed definition of severity and degree of injury

ii. The number of third and fourth degree tears as a percentage of annual vaginal deliveries

iii. The proportion repaired in the theatre, analgesia and suture materials used, method of repair and grade of operator

iv. Adequate note-keeping and counselling

v. The proportion seen at follow-up after postnatal period with symptom

vi. Questionnaire

Percentage continence rate following primary repair (cf $84 \%$ in our case, at 6 weeks follow-up).

\section{Results}

Total annual birth for 2003 was 2255 and for 2004 was 2329 . Total vaginal delivery for 2003 was 1771 and for 2004 was 1827 . Total number of women with major perineal tears for 2003-2004 were 61(53 had third degree tear and 8 had fourth degree tear).

According to the United Kingdom Census in 2001, total number of women in Barnsley was 111,977, the total number of women in the child bearing age group of 15 to 49 was 51,041 . Thus the prevalence of perineal trauma in Barnsley was 6.4/10,000 women $(32 / 51,041)$ for 2003 and 5.8/10,000 women (29/51,041) for 2004 (Tables 1-5).

Table I Age groups/gestational age

\begin{tabular}{llll}
\hline Age group & $\begin{array}{l}\text { Number of } \\
\text { women }\end{array}$ & Gestation(weeks) & $\begin{array}{l}\text { Number of } \\
\text { women }\end{array}$ \\
\hline$<20$ & 8 & $<37$ & 0 \\
$21-25$ & 18 & $37-40$ & 28 \\
$26-30$ & 15 & $>40$ & 33 \\
$31-35$ & 17 & & \\
$36-40$ & 3 & & \\
$>40$ & 0 & & 61 \\
Total & 61 & Grand total &
\end{tabular}

Table 2 Gravidity and parity/onset of labour/position of presenting part

\begin{tabular}{|c|c|c|c|c|c|c|c|}
\hline Gravidity & Total & Parity & Total & $\begin{array}{l}\text { Onset } \\
\text { of labour }\end{array}$ & Total & $\begin{array}{l}\text { Position } \\
\text { of part }\end{array}$ & Total \\
\hline I & 38 & 0 & 51 & spontaneous & 26 & $\mathrm{C}+\mathrm{OA}$ & 54 \\
\hline 2 & 15 & 1 & 9 & $\begin{array}{l}\text { Spontaneous } \\
\& \text { augmented }\end{array}$ & 21 & $\mathrm{C}+\mathrm{OP}$ & 6 \\
\hline 3 & 5 & 2 & I & induced & 14 & $\mathrm{C}+\mathrm{OT}$ & 1 \\
\hline 4 & 2 & & & & & & \\
\hline 5 & 0 & & & & & & \\
\hline 6 & 1 & & & & & & \\
\hline $\begin{array}{l}\text { Grand } \\
\text { total }\end{array}$ & 61 & & 61 & & 61 & & 61 \\
\hline
\end{tabular}


Table 3 Duration of labour/analgesia in labour

\begin{tabular}{llll}
\hline $\begin{array}{l}\text { Duration of 2nd stage of } \\
\text { labour (hrs) }\end{array}$ & Total & analgesia in labour & \\
\hline$<$ I & 19 & epidural & 27 \\
I-2 & 30 & diamorphine/pethidine & 22 \\
$2-3$ & II & entonox & 5 I \\
$>3$ & I & Tens & 8 \\
Grand total & 61 & & \\
\hline
\end{tabular}

Table 4 Type of delivery/weight of baby/episiotomy

\begin{tabular}{llllll}
\hline Type of delivery & Total & $\begin{array}{l}\text { Birth } \\
\text { weight(g) }\end{array}$ & Total & Episiotomy & \\
\hline Normal & 39 & $250 I-3000$ & 4 & yes & 30 \\
Ventouse & 8 & $300 I-3500$ & 19 & no & 31 \\
Forceps & 2 & $350 I-4000$ & 28 & & \\
Failed ventouse + Forceps & 12 & $>4000$ & 10 & & 61 \\
Grand total & 61 & & 61 & & \\
\hline
\end{tabular}

Table 5 Degree of tear/method of repair

\begin{tabular}{llll}
\hline Degree of tear & Total & Method of repair used & Total \\
\hline 3a & 6 & end-to-end & 28 \\
$3 \mathrm{~b}$ & 3 & overlap & 18 \\
$3 \mathrm{c}$ & 2 & not stated & 15 \\
3 Unclassified & 42 & & \\
4 th & 8 & & 61 \\
Grand Total & 61 & Grand total & \\
\hline
\end{tabular}

\section{Other findings were as follows}

Complications at or after delivery: at delivery: Postpartum haemorrhage (6), manual removal of retained placenta (3), shoulders dystocia (2), cord round the neck (1), compound presentation (1), dural tap (1) and others (4). After delivery: urine retention (1), anaemia/blood transfusion (2), failed spinal procedure (1), diarrhoea (1) Faecal/flatus incontinence (1), others (2)

Place of repair: Theatre (60), delivery room (1).

Anaesthetics used: Spinal (40), Epidural (14), Epidural plus local anaesthetics (6), Spinal plus local anaesthetics (1).

\section{Grade of operator:}
a) Consultant (13)
b) Registrar (41)
c) Senior Senior House Officer/Acting Registrar (5)
d) Senior House Officer (2)*

Grade of Supervisor:
A. Consultant (10)
B. Registrar $(2 *)$
C. Not supervised (49)
D. *Senior House Officers supervised by on- call Registrars

\section{Observations/symptoms at follow up 6-12 weeks after delivery:}

a. Rectal bleeding.

b. Some degree of faecal and flatus incontinence.

c. Feels suture material.

d. Leakage of faecal matter into the vagina.

e. Mild/moderate incontinence of flatus.

f. Urinary symptoms/constipation.

g. Sutures fell off/recto-vaginal fistula (confirmed by endoanal ultrasound scan).

h. Follow-up by colorectal surgeons (2 patients).

i. Did not attend follow up (11 patients).

j. No documentation about plan for 6 weeks post natal check. No record of any post natal visit (1 patient).

k. Continence rate at 6 weeks postnatal period was calculated as 42 patients at 6 weeks without symptoms divided by the total number of patients (50) who attended the post natal clinic, which was about $84 \%$.

Details of perineal tear: The Table 5 below illustrates the details of the perineal injury, the method of repair and the suture materials used:

\section{Discussion}

The incidence of major perineal tears in our unit was $1.8 \%$ (cf $0.6-$ $9 \%$ ) for 2003 and $1.56 \%$ (cf $0.6-9 \%$ ) for 2004. Thus our figures were within the national average. In Table 6, classification of repair/method of repair; 42 women out of $53(79.25 \%)$ who sustained third degree tear, the lesion was unclassified contrary to what was stipulated by the guidelines. Of the 61 repairs performed, the method of repair was not stated in 15 women $(24.59 \%)$.

Table 6 Suture materials used

\begin{tabular}{|c|c|c|c|c|c|c|c|}
\hline $\begin{array}{l}\text { External. } \\
\text { anal } \\
\text { sphincter }\end{array}$ & Total & $\begin{array}{l}\text { Internal } \\
\text { sphincter }\end{array}$ & Total & $\begin{array}{l}\text { Anal } \\
\text { canal }\end{array}$ & Total & Perineum & Total \\
\hline None & 0 & none & 51 & none & 53 & none & 10 \\
\hline PDS & 7 & PDS & 1 & $\begin{array}{l}\text { PDS } \\
3 / 0\end{array}$ & 1 & PDS $2 / 0$ & 1 \\
\hline PDS $2 / 0$ & 21 & PDS $2 / 0$ & 4 & $\begin{array}{l}\text { proline } \\
3 / 0\end{array}$ & 1 & vicryl & 26 \\
\hline PDS $3 / 0$ & 18 & PDS $3 / 0$ & 2 & vicryl & 1 & vicryl 2/0 & 21 \\
\hline vicryl & 2 & vicryl I/0 & 1 & $\begin{array}{l}\text { vicryl } \\
2 / 0\end{array}$ & 3 & vicryl $3 / 0$ & 1 \\
\hline vicryl I/0 & 2 & vicryl 2/0 & 2 & $\begin{array}{l}\text { vicryl } \\
3 / 0\end{array}$ & 2 & vicryl rapid & 2 \\
\hline vicryl $2 / 0$ & 7 & & & & & & \\
\hline vicryl $3 / 0$ & 4 & & & & & & \\
\hline $\begin{array}{l}\text { Grand } \\
\text { Total }\end{array}$ & 61 & $\begin{array}{l}\text { Grand } \\
\text { Total }\end{array}$ & 61 & $\begin{array}{l}\text { Grand } \\
\text { Total }\end{array}$ & 61 & $\begin{array}{l}\text { Grand } \\
\text { Total }\end{array}$ & 61 \\
\hline
\end{tabular}

In the results stated above in relation to the place of repair; 60 women $(98.36 \%)$ had their repair performed in the theatre. There was no breach of the guidelines on the anaesthetics used. In Table 6, suture materials used; the guideline advised use of PDS for the repair of the external and internal anal sphincters but in 15 women (24.59\%) vicryl 
was used to repair external anal sphincter. In 2 women, the type of vicryl used in repair was not stated. However, in 3 women, vicryl was used for the repair of internal anal sphincter. The strength of PDS was not stated in 7 women $(15.22 \%)$ out of the 46 women that had PDS used for the repair of external anal sphincter. 1 woman had internal anal sphincter repaired with PDS (strength of PDS not stated). 1 woman had anal sphincter repaired with PDS $3 / 0$ and one woman had perineal repair with PDS 2/0.

In Table 7, use of antibiotics; in 30 women ( $50 \%$ ) out of 60 , no intraoperative antibiotic was given contrary to advice from the guideline but 59 women $(96.72 \%)$ had oral postoperative antibiotics. In 2 women $(3.28 \%)$ there was no documentation of any antibiotic given postoperatively. It could not be ascertained whether the 30 women who did not have intraoperative antibiotics had more complications such as infection than those who had.

Table 7 Intravenous intraoperative/oral post-operative antibiotics

\begin{tabular}{|c|c|c|c|}
\hline Intra-operative & Total & Postoperative & Total \\
\hline Augmentin \& Metrinidazole & 4 & Cephradine \& Metronidazole & 58 \\
\hline Augumetin only & 6 & Erythromycin \& Metronidazole & 1 \\
\hline $\begin{array}{l}\text { Cefuroxime \& } \\
\text { Metronidazole }\end{array}$ & 20 & Not Documented & 2 \\
\hline Clindamycin (for GBS) & 1 & & \\
\hline Not Documented & 30 & & \\
\hline Grand Total & 61 & Grand Total & 61 \\
\hline
\end{tabular}

GBS, group B streptococcus

In the stated results above, post-delivery complications; 51 women $(83.61 \%)$ out of 61 had no documented complications and 8 had complication as stated. Some of the complications such as diarrhoea may be related to medications such as use of laxatives/antibiotics or to anaesthetic medication used, while some such as anaemia, haematuria/ urine retention were likely to be related to the main delivery event rather that the repair itself.

Table 8, adequate note keeping; in all the women's anatomical structures were stated. Method of repair was stated only in 46 women $(75.41 \%)$ out of 61 . In 19 women $(31.15 \%)$ swabs/sharps/ instruments were not documented to have been counted. This may raise medico-legal issue(s). In 36 women $(59.02 \%)$ out of 61 , there were no illustrative diagrams of the injury sustained. In Table 9, other management; 25 women (40.98\%) had no documented counselling. 11 women $(18.03 \%)$ were not consented for the procedure which again may raise medico-legal issue(s).

Table 8 Intravenous intraoperative/oral post-operative antibiotics

\begin{tabular}{lll}
\hline & Documented & $\begin{array}{l}\text { Not } \\
\text { documented }\end{array}$ \\
\hline Anatomical Structure Involved & 61 & 0 \\
Method of Repair & 46 & 15 \\
Suture Material Used & 61 & 19 \\
Instruments/Swabs/Sharp count & 42 & 36 \\
Diagram of Injury & 25 & \\
\hline
\end{tabular}

GBS, group B streptococcus
Table 9 Intravenous intraoperative/oral post-operative antibiotics

\begin{tabular}{lll}
\hline & Documented & $\begin{array}{l}\text { Not } \\
\text { documented }\end{array}$ \\
\hline Counselling & 36 & 25 \\
Consented & 50 & 11 \\
Rectal Examination & 47 & 14 \\
Vaginal Examination & 47 & 14 \\
Laxatives/Bulking Agents & 61 & 0 \\
Bowel Opened Before Discharge & 61 & 0 \\
\hline
\end{tabular}

GBS, group B streptococcus

14 women $(22.95 \%)$ have no documented vaginal/rectal examination. All women had laxatives postoperatively and all had their bowels opened before discharge from the hospital. In Table 10, follow-up (6weeks/12weeks); 60 women (98.36\%) had documented booking for follow-up. 50 women (81.97\%) out of 61 had attended the follow-up care. All the attendees had completed symptom questionnaire documented.

Table 10 Follow-up at 6-12 weeks postnatal period

\begin{tabular}{lll}
\hline Booked for consultant care & 60 & I not documented \\
\hline Attended & 50 & II not attended \\
Documented Symptoms Questionnaire & 50 & II none \\
Symptoms Documented & 42 no & $\begin{array}{l}7 \text { had symptoms } \\
\text { related/unrelated }\end{array}$ \\
& symptoms & I not documented \\
Endoanal Scan & & 58 did not have it \\
Findings on Scan & 2 had it & \\
Follow up Required & 2 had \\
significant & findings & \\
\hline
\end{tabular}

42 women $(84 \%)$ out of 50 who had attended had no symptoms while 8 women $(16 \%)$ had various related/unrelated symptoms. Only 2 women $(4 \%)$ out of 50 who attended needed an endoanal scan and the same 2 women (4\%) needed follow-up beyond the 6 week/12week time frame. The guideline advised longer follow-up beyond this time frame because sphincter problems following delivery can appear several months after delivery.

The risk factors ${ }^{1}$ for the major perineal tears from retrospective studies are as follows in the order of severity of the risk factors; the use of forceps for delivery carry a risk of 7\%; nulliparity and second stage of labour lasting over 1 hour, each carry a risk of $4 \%$; episiotomy and persistent occipito-posterior position, each carry a $3 \%$ risk while induction of labour, birth weight over $4 \mathrm{~kg}$ and epidural analgesia, each carry a risk of $2 \%$. However, it is important to note that the knowledge of identified risk factors cannot prevent major perineal tears and some of these factors cannot be prevented e.g. nulliparity, use of epidural and induction of labour.

The prevalence of symptoms after repair was $25-57 \%$ according to the RCOG meta-analysis ${ }^{1}$ and in another study, ${ }^{2}$ there was increase $(17-42 \%)$ in anal incontinence on long term follow-up of about 4 years. The symptoms following primary repair in the literature 1 were similar to the findings of this study at 6-12 weeks follow-up shown above. 
This study showed that some areas of the management of these women were in line with the evidence-based RCOG guidelines and some areas were non-compliant with or had different approach to the guidelines. There were management and note keeping issues which according to the author may have medico-legal significance. The last study in 2003, essentially a review, did not compare our practice with the standard practice and did not state the continence rate (part of auditable standards). The author was therefore unable to comment on any improvement since the last study in relation to the definitions of perineal injury and relevant provisions of clinical governance. The last study in 2003 however, had recommended classification of third degree tear. This was not done in the 42 women (79.25\%) as shown by the present study. It was also noted that the operational local protocol which came into effect in 2004 appeared not to have embraced the RCOG guideline of 2001 on the classification of third degree perineal tear.

In the studies mentioned in the RCOG meta-analysis, endoanal ultrasound was used in the follow-up of these patients and demonstrated persistent sphincteric defects of $54-58 \%$ but the clinical relevance of asymptomatic cases has so far remained unclear. ${ }^{1}$ A study in the USA ${ }^{5}$ showed greater incidence of perineal tears (17\%), higher than the figures seen in the United Kingdom, while other studies, ${ }^{6-8}$ attributed this high incidence to the frequent use of a median episiotomy in the USA. A Swedish study ${ }^{9}$ recommended that median episiotomy should be avoided.

A US study ${ }^{10}$ showed that a previous third degree tear was a predictor of further major tears and was associated with 3.4 fold increased risk. However, another study ${ }^{11}$ disagreed with this and stated that operative vaginal delivery and episiotomy were the main risk factors for recurrence of tears at subsequent vaginal delivery. Another study ${ }^{12}$ concluded there were still variations in management of obstetric tears and choice of subsequent mode of delivery among obstetricians.

\section{Recommendations}

1. The local protocol should be reviewed in line with the findings of this study and also with the view of encouraging classification of third degree tears as stated in RCOG guidelines of 2001.

2. Articulate graded/step by step summary of management of repair by way of concise graphic illustrations can be placed in the labour suite and theatre to remind staff what they should do in third and fourth degree repair. This could enhance compliance with the guidelines especially with respect to note keeping.

3. Appropriate classification of third degree tear is important because the three types of $3 \mathrm{rd}$ degree tear have prognostic and medico-legal significance.

4. Use of appropriate suture materials as stipulated in the guidelines should be adhered to.

5. The postnatal follow-up should be homogenized to take place earlier at 6 weeks postnatal period (not at 3 months after delivery), to assess continence rate at 6 weeks and discuss other issues or concerns. The author recommended a further visit in Gynaecologic out patients in 6-12months time for a further review. This would give more time for any latent anal sphincter symptoms to become manifest and be attended to.

6. In view of the nature of the major perineal injuries and its medico legal significance, it is advisable for trainees to attend a perineal trauma course prior to performing repairs direct supervision. This would enable trainees to refresh their understanding of the physiology and anatomy of the anal sphincter and the mechanics of the injury during child birth.

7. According to the definition/provisions of clinical governance, this audit should be a yearly rolling audit to see if there is improved compliance with the guidelines on management/note keeping.

8. It is essential that patients are counselled and consented for the procedure whenever possible and where not possible, this should be done after the procedure.

9. Intravenous intraoperative antibiotics are recommended to prevent early onset of infection. This is because it takes a while before these women start their oral antibiotics which in turn have to undergo first pass metabolism before becoming active in vivo. The time lag before oral antibiotic become active meant that infection can develop before the effect of oral antibiotic takes hold.

10. Anal endosonography after delivery allows the diagnosis of clinically undetected anal sphincter injury ${ }^{13}$ and its routine application may not be appealing in view of costs but there may be a case to make it optional and could be offered on request.

11. Though the provisions of our local operational protocol was very condensed on this subject (pages 30-32 of the protocol), and needs some elaboration, all efforts should be made to adhere to the protocol till it have been updated in line with new evidence.

\section{Acknowledgments}

Information Technology Team, Clinical Audit Department and Library staff, Barnsley Hospitals NHS Foundation Trust for technical and other support.

\section{Conflicts of interest}

The authors declare there is no conflict of interests.

\section{References}

1. RCOG. RCOG Green-top Guideline 29. London; 2001.

2. Poen AC, Felt-Bersma RJ, Strijers RL, et al. Third degree obstetric perineal tear: Long term clinical and functional results after primary repair. Br J Surg. 1998;85(10):1433-1438.

3. RCOG. RCOG Green-top guideline 23. London; 2004.

4. Fitzpatrick M, Behan M, O’Connell PR, et al. A randomised clinical trial comparing primary overlap with approximation (end to end) repair of third degree obstetric tears. Am J Obstet Gynecol. 2000;183(5):12201224.

5. Legino LT, Woods MP, Rayburn F, et al. Third and fourth-degree perineal tear: 50 years' experience at a university hospital. J Repro Med. 1998;33(5):423-426.

6. Golden WE, Sanchez N. The relationships of episiotomy to third and fourth degree lacerations. J Ark Med Soc. 1996;92(9):447-448.

7. Klein MC, Gauthier RJ, Robbins JM, et al. Relationship of episiotomy to perineal trauma and morbidity, sexual dysfunction, and pelvic floor relaxation. Am J Obstet Gynecol. 1994;171(3):591-598.

8. Labrecque LT, Baillageon L, Dallaire M, et al. Association between median episiotomy and severe perineal laceration in primiparous women. CMAJ. 1997.156(6):797-802. 
9. Jander C, Lyrenas S. Third and Fourth degree perineal tears: Predictor factors in a referral Hospital. Acta Obstet Gynecol Scand. 2001;80(3):229-234.

10. Payne TN, Carey JC, Rayburn WF. Prior third or fourth degree perineal tear and recurrence risks. Int J Gynaecol Obstet. 1999;64(1):55-57.

11. Dandolu V, Gaughan JP, Chatwani AJ, et al. Risk of recurrence of anal sphincter lacerations. Am J Obstet Gynecol. 2005;105(4): 831-835.
12. Ononeze BO, Gleeson N, Turner MJ. Management of third and fourth degree tear and choice of mode of delivery in subsequent pregnancies. $J$ Obstet Gynaecol. 2004;24(2):148-151.

13. Faltin DL, Boulvain M, Irion O, et al. Diagnosis of anal sphincter tears by postpartum endosonography. Am J Obstet Gynecol. 2000;95(5):643-647. 